\title{
Bericht über die Tagung Globales Lernen zwischen politischem Bildungsauftrag, Apokalypse, Klimagerechtigkeit und Handlungsdruck
}

Am 01./02.10.2019 trafen sich in der Alten Feuerwache Berlin-Kreuzberg 65 Teilnehmer/-innen aus NGOs, Schule, Verwaltung und Wissenschaft. Leitend für die Tagung war die Frage, welche Antworten Globales Lernen (GL) und Bildung für nachhaltige Entwicklung (BNE) angesichts der dramatischen Folgen des Klimawandels auf die Frage einer gerechten Zukunft für die Weltgesellschaft in einer postfossilen Ära finden können. Und worum es gehen kann, wenn wir in Bildung und Schule von einer großen Transformation sprechen.

Die Tagung eröffnete Dr. Mandy Singer-Brodowski vom Institut Futur der Freien Universität Berlin mit ihrem Vortrag Lernen durch Klimakrise, große Transformation und globale Gerechtigkeit. Sie wies eingangs darauf hin, dass wir uns schon inmitten von Klimakrise und großer Transformation befinden. Bezugnehmend auf das Gutachten des Wissenschaftlichen Beirats für Globale Umweltveränderung beschrieb Singer-Brodowski die große Transformation als einen tiefgreifenden Wandel von Infrastruktur und Produktionsprozessen, Regulierungssystemen und Lebensstilen, flankiert von einem neuen Zusammenspiel von Wissenschaft, Gesellschaft, Wirtschaft und Politik. Sie verwies auf das Spannungsverhältnis eines dafür notwendigen starken Staates und einer starken $\mathrm{Zi}$ vilgesellschaft. Bemerkenswert war in diesem Kontext der historische Blick auf internationale Nachhaltigkeitspolitik. Während in den letzten Jahrzehnten in der Wirtschaft ein Paradigmenwechsel von der Wirtschaft gegen die Umwelt zu Wirtschaftshandeln für die Umwelt stattfand, erfolgte in der Politik der Rückzug staatlicher Steuerung hin zu eher marktbasierten Ansätzen. Währenddessen verschob sich der anfangs radikale gesellschaftliche Diskurs über die Grenzen des Wachstums hin zu einem technokratischen Diskurs, der mit grünem Wachstum Nachhaltigkeitsziele erreichen will. Die Vortragende beschrieb in diesem Spannungsfeld die Notwendigkeit, dass BNE und GL bei der erfolgreichen Implementierung in Schulen, Hochschulen und Lehrplänen immer wieder reflektieren müssen, inwieweit kritische Perspektiven sichtbar werden können. Dabei problematisierte sie den Zwang von Bildungsakteur/-innen, ihre Angebote anschlussfähig an die politischen Rahmenbedingungen $\mathrm{zu}$ machen und ihre eigene Kapitalismus-, Wachstums- und postkoloniale Kritik etwas in den Hintergrund zu stellen. Gerade in den jüngeren Diskussionen seien eine unkritische Annahme von Wirtschaftswachstum als einzigem Paradigma sowie ein sehr utilitaristischer Zugriff auf Natur und Ökosysteme zu verzeichnen. Sie empfahl, subversives Lernen über die Treiber der Klimakrise sehr viel mehr in den Mittelpunkt zu stellen, Wachstumsfragen

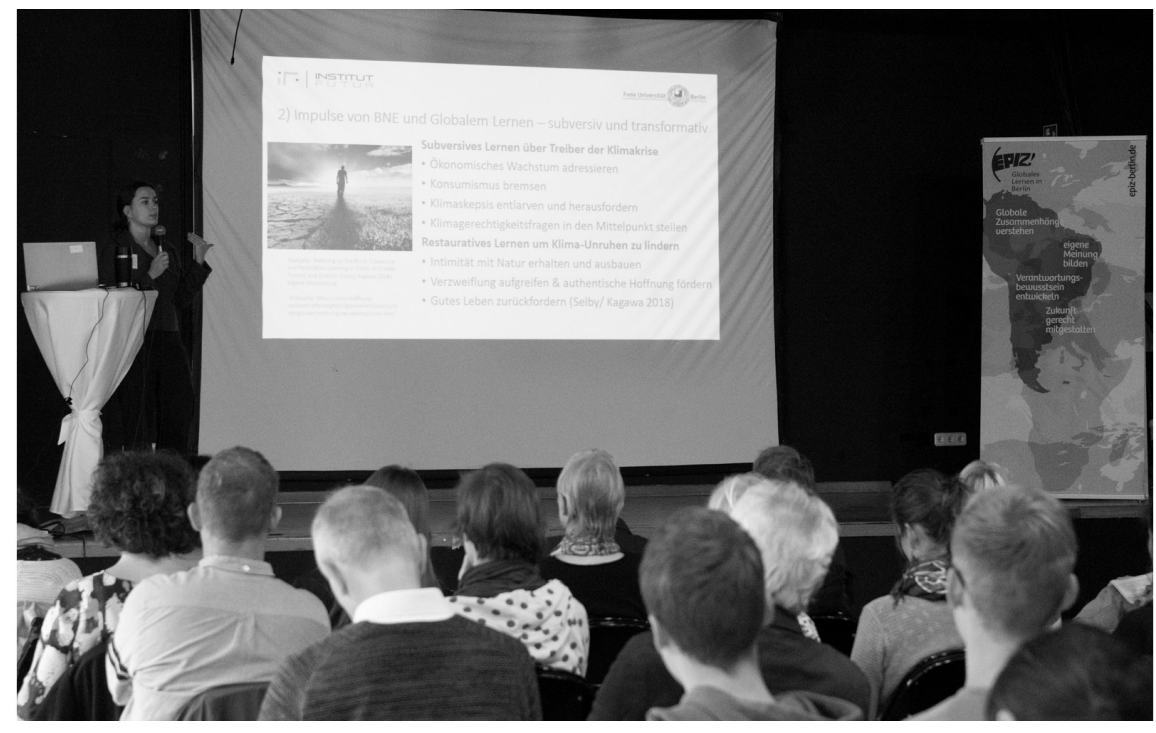

Abb. 1: Dr. Mandy Singer-Brodowski (Institut Futur der Freien Universität Berlin) sprach zum Thema "Lernen durch Klimakrise, große Transformation und globale Gerechtigkeit", Quelle: Mimoza Elgt, Jammin Photostudio \& Gallery Berlin 
stärker zu adressieren und wirtschaftliches (grünes) Wachstum zumindest als umstrittenes Feld in der Nachhaltigkeitscommunity zu hinterfragen.

Im weiteren Tagungsverlauf fanden insgesamt sieben Workshops statt, die politische und pädagogische Herausforderungen von schulischen Akteur/-innen und von Zivilgesellschaft angesichts von Klimakrise und politischem Handlungsdruck aufgriffen. Der Workshop Nachhaltiger Klimaschutz im Spannungsfeld von Staat und Zivilgesellschaft mit Kai Bergmann von Germanwatch e.V. diskutierte Rollen, Spielräume und Strategien zivilgesellschaftlicher Institutionen. Aus systemtheoretischer Perspektive wurde die komplexe Eigenlogik sozialer Teilsysteme diskutiert, die Möglichkeit „disruptiver Veränderungen" in einer sich polarisierenden Gesellschaft ausgelotet und auf eigene Handlungsoptionen im Sinne von „Vergrößere Deinen Handabdruck, verringere Deinen Fußabdruck!“ fokussiert. Im Workshop Jugendprotest, Klimakrise und Selbstermächtigung mit dem Journalisten Rico Grimm von den Krautreportern wurden ebendiese Fragen dezidiert aus der Perspektive der Protestbewegung Fridays for Future aufgegriffen. Deren Aktionsformen, ihr Generationenverhältnis wie auch Bezüge zur Schule warfen spannende Fragen - z.B. wie die nach zu erwartenden Radikalisierungsprozessen angesichts der aktuellen Klimaschutzpolitik - auf. Im Mittelpunkt standen dabei die Entstehungsbedingungen und politischen Forderungen der Bewegung, deren Klimakommunikationsformen sowie ihr Verhältnis zu anderen politischen Akteur/-innen. Der Workshop Dekoloniale Perspektiven auf transformatives Lernen mit Abdou Rahime Diallo vom Netzwerk Migrantenorganisationen Brandenburg e.V. zielte darauf, globale Herausforderungen und Prekaritäten auf Wirkzusammenhänge des Kolonialismus zu prüfen. Die Teilnehmer/-innen des Workshops diskutierten, inwieweit sich Konzepte transformativer Bildung und Globalen Lernens von eurozentristischen Paradigmen emanzipieren können, Haltungen, Werte und Perspektiven aus dem politischen Globalen Süden berücksichtigt werden, und auf welchen Wegen eine tiefgründige Dekonstruktion und Dekolonisierung von Wissens- und Lernarealen erfolgen kann. Der Workshop Transformative Bildung inklusiv hingegen mit Katarina Roncevic aus dem Bildungsteam von Greenpeace eruierte mit einem weit gefassten Inklusionsbegriff, wie der An- spruch Globalen Lernens - niemanden in den Bildungsinstitutionen zurückzulassen - in der Schule inklusiv im Sinne eines Whole School Approaches mit konkreten Ideen umgesetzt werden kann. Im Workshop Mit Medienkompetenz gegen Klimawandelleugnung wurde das vom EPIZ neu entwickelte Konzept zur Medienkompetenz anhand von Klimawandelnachrichten vorgestellt und dessen Möglichkeiten und Grenzen diskutiert. Der Workshop Globales Lernen in ländlichen Strukturen reflektierte wiederum die Frage, wie entwicklungspolitische Bildungsangebote konzipiert werden müssten, um die Lebenswirklichkeiten in ländlichen Räumen zu treffen. Stephanie Günther, Fachpromotorin für GL in Brandenburg, stellte zahlreiche Herausforderungen für GL in ländlichen Räumen vor: angefangen bei infrastrukturellen Fragen über ländliche Bündnispartnerschaften bis hin zu spezifischen Kommunikationsstrategien zur Implementierung globaler Themen. Das Thema Ressourcengerechtigkeit und Postwachstumsökonomie wurde im Workshop mit Nele Cölsch von Fairbindung e.V. aufgegriffen. Angesichts der Fixierung auf das Wachstum des Bruttoinlandprodukts und der Tatsache, dass unser global expandierendes Wirtschaftsmodell die ökologische Tragfähigkeit des Planeten bei weitem sprengt, diskutierten die Teilnehmenden den Gewinn von an Postwachstum und Gemeinwohl orientierten Ansätzen zur Lösung der sozialen und ökologischen Krisen.

Bleibt noch zu erwähnen, dass der zweite Konferenztag mit der Podiumsdiskussion „Zivilgesellschaftlicher Protest, Politik und Schule - was muss passieren, damit etwas passiert?" begann. Auf dem Podium diskutierten Linda Volker, Sprecherin von Fridays for Future Berlin, Corina Conrad-Beck von der Senatsverwaltung für Umwelt, Verkehr und Klimaschutz Berlin sowie Moritz Voges als Fachpromotor für Globales Lernen aus Berlin. Das Podium wie auch die gesamte Tagung moderierte Dr. Christina Ayazi von der Sigmund-Freud-PrivatUniversität Berlin. Eine Dokumentation der Tagung ist in Arbeit. Eine ausführliche Dokumentation der Tagung erfolgt durch die Organisator/-innen der Tagung vom EPIZ Berlin und ist in Kürze auf deren Website verfügbar.

Matthias Schwerendt, EPIZ Berlin e.V. doi.org/10.31244/zep.2020.01.10 